\title{
Die Akademie für Fort- und Weiterbildung in der Radiologie informiert
}

Kontinuierliche Fortbildung ist für Sie als Arzt oder Ärztin und auch als MTRA selbstverständlich, um eine gute Patientenversorgung sicherzustellen. Die Akademie für Fort- und Weiterbildung in der Radiologie unterstützt Sie dabei. Gemeinsam mit vielen Partnern bietet sie Ihnen ein umfangreiches Fort- und Weiterbildungsangebot, dessen Qualität durch fortwährende Evaluation gewährleistet wird.

\section{Kennen Sie schon unseren interak- tiven Fortbildungskalender? \\ $\nabla$}

Um Ihnen die Auswahl der für Sie interessantesten Fortbildungsveranstaltungen zu erleichtern, bieten wir Ihnen mit unserem interaktiven Veranstaltungskalender eine Übersicht über eine Vielzahl von Veranstaltungen aus dem Bereich der Radiologie. Sie finden Präsenzveranstaltungen im In- und Ausland, aber auch zeitsparende Online-Kurse für alle Fachrichtungen. Sie sehen sofort wie viele Fortbildungspunkte Sie mit Ihrer Teilnahme erhalten. Mit einem Klick können Sie die Veranstaltungsdaten direkt in Ihren eigenen Kalender übernehmen oder sich sofort zu der gewünschten Veranstaltung anmelden.

Darüber hinaus bietet Ihnen unser interaktiver Veranstaltungskalender die Möglichkeit, eine persönliche Auswahl von Veranstaltungen nach bestimmten Kriterien wie z. B. Veranstaltungsort, Zeitraum, Thema oder Zielgruppe zu erstellen. So finden Sie gewünschte Veranstaltungen in wenigen Sekunden.

Überzeugen Sie sich selbst unter www. drgakademie.de - Veranstaltungskalen$\operatorname{der}(\odot$ Abb. 1).

Sie möchten tagesaktuell über die für Sie interessanten Fortbildungsveranstaltungen informiert werden? $\nabla$

Nutzen Sie unseren neuen Service, sich im Mitgliederbereich ein persönliches Profil für die Planung Ihrer Fortbildungstermine anzulegen. Setzen Sie Ihre eigenen Schwerpunkte und Sie erhalten immer

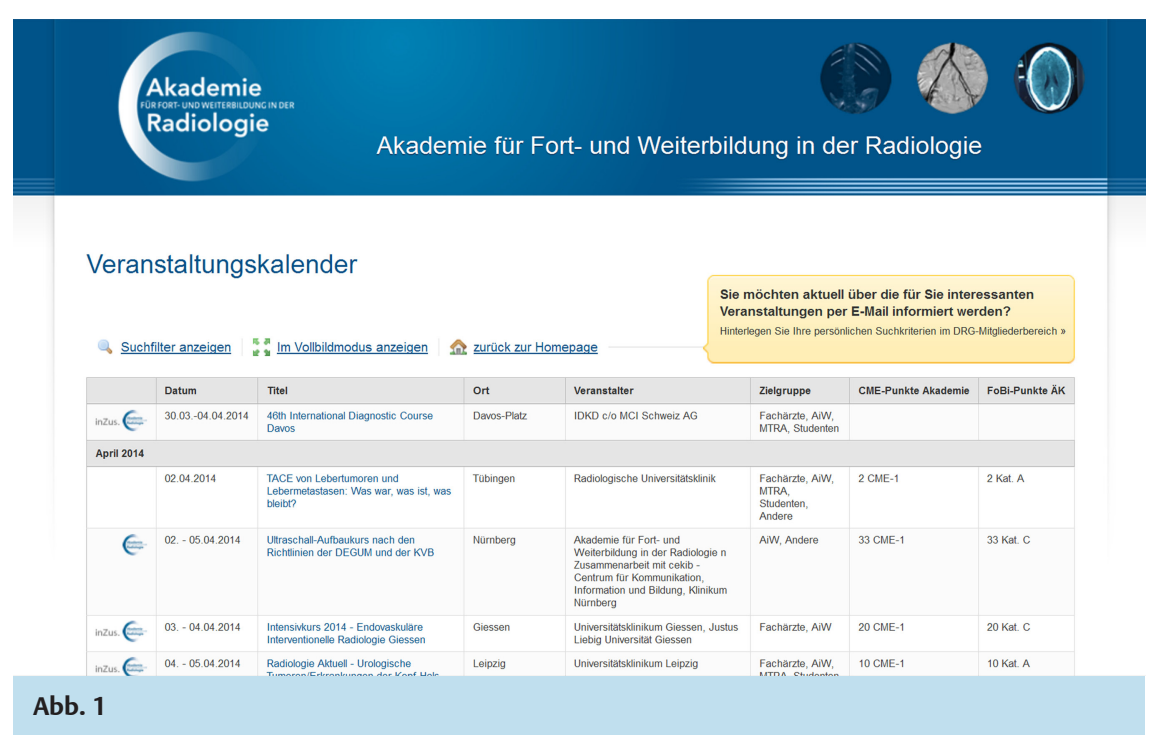




\section{Was müssen Sie dafür tun?}

$\nabla$

Tragen Sie Ihre Auswahlkriterien, wie z.B. Veranstaltungsort, Zielgruppe und/oder Thema und Ihre E-Mail-Adresse in Ihr Suchprofil ein und Sie erhalten von uns in einem persönlichen Newsletter zeitnah aktuellste Informationen, ausgewählt aufgrund Ihrer individuellen Schwerpunkte. Gleichzeitig geben wir Ihnen die Möglichkeit, die Termine direkt in Ihren persönlichen Kalender einzutragen.

Selbstverständlich ist eine Aktualisierung Ihrer Auswahlkriterien jederzeit möglich.

Testen Sie am besten gleich selbst unseren neuen Service! Loggen Sie sich im Mitgliederbereich der DRG ein unter www.drg. de/de-DE/744/login und legen Sie sich Ihr Suchprofil an (๑ Abb.2).

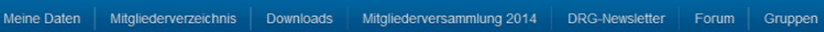

Start = DRG Login = Meine Daten = Suchprofil

Mein Suchprofil für Fortbildungsveranstaltungen

Sie möchten immer aktuell informiert werden, wann und wo die für Sie interessanten Fortbildungsveranstaltungen stattfinden? Mit dem Suchprofil können Sie Veranstaltungsinformationen gezielt per Mail abonnieren.

Kombinieren Sie Ihre Auswahlikrterien wie Veranstaltungsort. Zielgruppe und Themen und erhalten Sie tagesaktuell Informationen uber neu in unserem Veranstaltungskalender verofftentlichte Veranstattungen per E-Mail von uns. Sie können Ihre Auswankikrterien jederzet ändern oder Ihr Suchprofil wieder loschen.

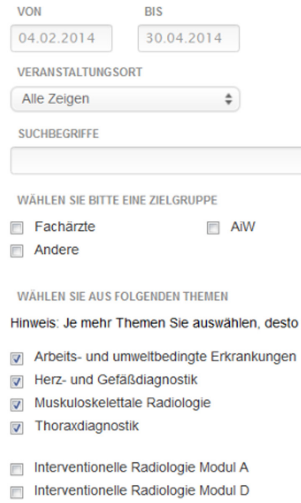

I MTRA

[ Ingenieure/ Naturwiss. $\square$ Studenten

Abb. 2

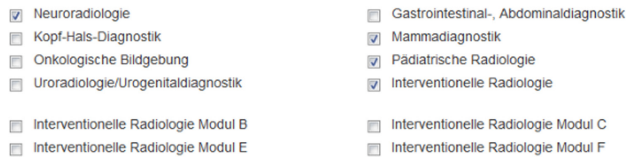

